\title{
The Role of Diamines in the Formation of Graphene Aerogels
}

\section{Katerina Vrettos ${ }^{1}$, Niki Karouta ${ }^{2}$, Panagiotis Loginos ${ }^{1}$, Suraj Donthula ${ }^{3}$, Dimitrios Gournis ${ }^{2}$ and Vasilios Georgakilas ${ }^{1 *}$}

${ }^{1}$ Material Science Department, University of Patras, Rio Patras, Greece, ${ }^{2}$ Department of Materials Science and Engineering, University of loannina, loannina, Greece, ${ }^{3}$ Department of Chemistry, Missuri University of Science \& Technology, Rolla, MO, United States

Aliphatic or aromatic diamines undergo nucleophilic attack on the epoxy groups of graphene oxide under hydrothermal conditions resulting in partial functionalization and partial reduction of the graphenic surface. The overall reaction decreases the solubility of graphene oxide and yields a hydrogel that can be dried to a 3D porous structure classified as an aerogel. This article compares the graphene aerogels derived from different aliphatic and aromatic diamines.

Keywords: graphene aerogels, diamines, conductive aerogels, carbon superstructures, porous nanostructures

\section{OPEN ACCESS}

Edited by:

Emilia Morallon

University of Alicante, Spain

Reviewed by:

Horacio Javier Salavagione

Consejo Superior de Investigaciones Científicas (CSIC), Spain

Tatiana S. Perova,

Trinity College, Dublin, Ireland

*Correspondence:

Vasilios Georgakilas viegeorgaki@upatras.gr

Specialty section:

This article was submitted to Carbon-Based Materials, a section of the journal

Frontiers in Materials

Received: 05 February 2018 Accepted: 22 March 2018 Published: 10 April 2018

Citation:

Vrettos $K$, Karouta N, Loginos $P$,

Donthula S, Gournis D and Georgakilas V (2018) The Role of

Diamines in the Formation of

Graphene Aerogels.

Front. Mater. 5:20.

doi: 10.3389/fmats.2018.00020

\section{INTRODUCTION}

Graphene Oxide (GO) is a graphene derivative with significant scientific interest that has been expanded enormously after the isolation of single layered graphene nanosheets by Novoselov et al. (Novoselov et al., 2004; Compton and Nguyen, 2010; Kim et al., 2010; Chen et al., 2012; Zhu et al., 2012). GO is essentially a graphene monolayer functionalized heavily with oxygen groups, mainly epoxides, hydroxyls, and carboxylates, although several others have been also proposed (Hontoria-Lucas et al., 1995; He et al., 1998; Lerf et al., 1998). GO is produced via liquid exfoliation of graphite oxide by treatment of graphite with strong acids according to established procedures by Staudenmaier (1898), Hofmann and Konig (1937), Hummers and Offeman (1958) that followed the first synthesis of GO by Brodie (1859), and its variants (Park and Ruoff, 2009; Mao et al., 2012). Although the GO core consists of hydrophobic aromatic domains, the presence of surface oxygen groups adds hydrophilic character that induces a characteristic colloidal stability of GO nanosheets in water. Successful reductive removal of those oxygen groups, and partial reconstruction of the aromatic system of GO results to the known-as reduced GO (rGO), which is receiving much attention as an alternative to pure graphene due to its good electrical properties (Pei and Cheng, 2012; Chua and Pumera, 2014). In addition, removal of oxygen groups reduces the hydrophilic character of rGO and results in aggregation in water. Thus, under appropriated conditions, the reductive aggregation of GO may give hydrogels, which can then be transformed to aerogels by removing pore filling water according to well-established procedures (e.g., freeze drying). Thereby, graphene aerogels are 3D architectures consisting of a conducting network of rGO nanosheets with large specific surface areas and micro/nano porosity (Li and Shi, 2012, 2014; Pei and Cheng, 2012; Zhang et al., 2012; Nardecchia et al., 2013; Li et al., 2014). Graphene aerogels are attracting much attention for applications in catalysis, environmental remediation, energy storage (supercapacitors, $\mathrm{Li}$ ion batteries) as well as in sensors and biosensors (Chen et al., 2013; Hu et al., 2013; Sun et al., 2013; Tang et al., 2014). Usually, graphene gelation is assisted by hydrophilic polymers or small organic molecules, although rGO hydrogels without such promoters have also been reported (Liu and Seo, 2010). The role of those promoters and their influence in the properties of the final product 


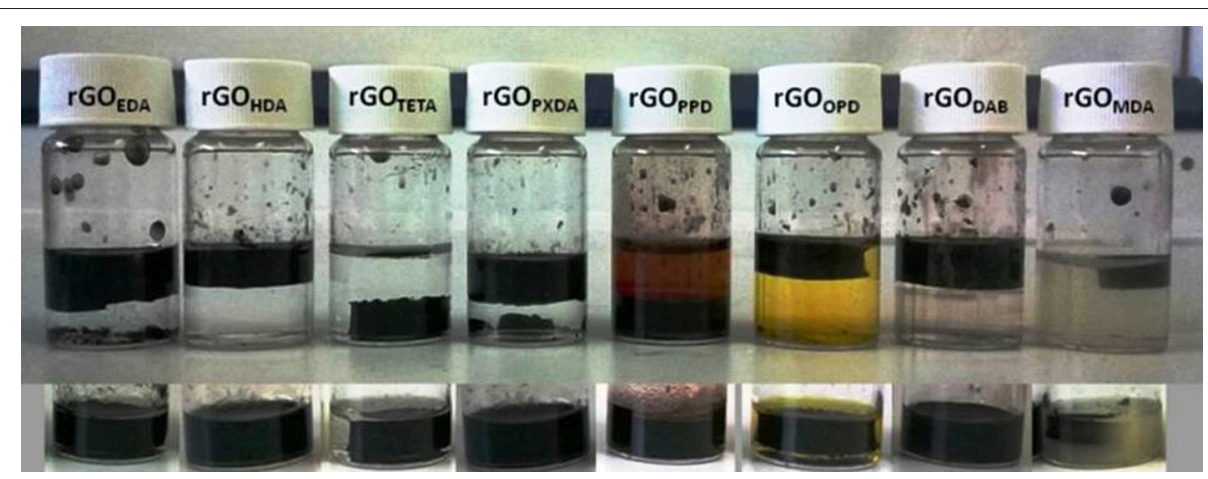

FIGURE 1 | Hydrogels as prepared by hydrothermal treatment (lower), and after the addition of water (upper).

has not been studied systematically. One particular class of such promoters, organic diamines, can act effectively as nucleophiles, attacking the epoxy groups of GO. In that regard, they get bonded on the surface of GO covalently, effectively bridging (cross linking) graphene sheets. Furthermore, diamines, being well-known reducing agents, become responsible for partial reconstruction of the aromatic system on rGO.

In view of the aforementioned, this study describes the preparation and characterization of a series of graphene aerogels derived with several aliphatic and aromatic diamines (aniline derivatives) as shown in Figure 1. Those diamines were selected based on their solubility in water, their molecular size and flexibility, their number of nitrogen atoms, their basicity, and their nucleophilicity. The effectiveness of those diamines as gelation promoters was investigated via the morphology and other material properties of the final products (aerogels).

\section{EXPERIMENTAL SECTION}

\section{Materials}

1,4-Phenylenediamine (Merck Millipore Schuchard), methylene dianiline (Chem-Cruz), 3,3-diaminobenzidine (FluoroChem), triethylenetetramine (Struers), 1,6 hexanediamine (Acros Organincs Inc.) 1,2- phenylenediamine (Sigma Aldrich), paraxylylenediamine (Fluka Chemicals) were used as received. Nitric acid (65\%), sulfuric acid (95-97\%), potassium chlorate, and powder graphite (purum) were acquired from Fluka.

\section{Synthesis of Go}

GO was prepared according to Staudenmaier's method (Staudenmaier, 1898) through oxidation of graphite. In a typical procedure, graphite $(5 \mathrm{~g})$ was added to a cold mixture of $200 \mathrm{~mL}$ $\mathrm{H}_{2} \mathrm{SO}_{4}$ and $100 \mathrm{~mL} \mathrm{HNO}_{3}$, in an ice-water bath. $\mathrm{KClO}_{3}$ powder $(100 \mathrm{~g})$ was added to the cold mixture in small portions under continuous stirring. The reaction was quenched after $20 \mathrm{~h}$ by pouring the mixture into distilled water. The oxidation product was washed until the $\mathrm{pH}$ reached 6.0, and was dried at room temperature.

\section{Synthesis of Graphene Aerogels}

GO $(12 \mathrm{mg})$ was dispersed in alkaline water $(4 \mathrm{~mL}+60 \mu \mathrm{L}$ of conc. aq. ammonium hydroxide) using ultrasonication for $1 \mathrm{~h}$. After addition of diamine $(0.1 \mathrm{mmol})$, the mixture was heated in a sealed bottle at $95^{\circ} \mathrm{C}$ for $24 \mathrm{~h}$. The resulting hydrogels were washed several times with water to remove unreacted diamine, and then they were lyophilized for $24 \mathrm{~h}$. The density of final aerogels was determined from their weight and their physical dimensions. (Hu et al., 2013; Tang et al., 2014).

\section{Materials Characterization}

Thermogravimetric analysis (TGA) was carried out with a TA Instrument Q500 Thermo Gravimetric Analyzer under ambient air with a heating rate of $10^{\circ} \mathrm{C} \mathrm{min}^{-1}$ up to $700^{\circ} \mathrm{C}$. Scanning electron microscopy (SEM) images was carried out on a Zeiss EVO-MA10, a Hitachi S-4700 field-emission microscope. FTIR spectra were obtained with a ATR technique on a FTS 3000 Excalibur Series Digilab spectrometer and on a Shimadzu FT-IR 8400 spectrometer equipped with a deuterated triglycine sulfate detector. The samples were in the form of $\mathrm{KBr}$ pellets containing ca. $2 \% \mathrm{w} / \mathrm{w}$ of the material.

XRD was conducted with a D8 Advance Bruker diffractometer using a CuKa (ID1.5418) radiation source $(40 \mathrm{kV}, 40 \mathrm{~mA})$ and a secondary beam graphite monochromator. Diffraction patterns were recorded in the 2-theta (2') scale from $2^{\circ}$ to $80^{\circ}$, in steps of $0.02^{\circ}$ and with a counting time of $2 \mathrm{~s}$ per step. Raman spectra were collected with a Raman system LabRam HR Evolution RM (Horiba-Scientific) using a laser excitation line at $532 \mathrm{~nm}$ (laser diode). The laser power was $1.082 \mathrm{mV}$. All Raman parameters have carefully controlled to avoid changes in the graphene materials. Bulk resistance was measured using a Keithley 2401 multimeter with two ITO glasses as electrodes that covered the upper and lower surface of the aerogels. The conductivity was estimated taking into consideration the surface of the aerogel as shown in Table 2 and the thickness $1=3 \mathrm{~mm}$.

The porosity of the aerogels was estimated by the following equation $\varphi=V_{\text {pore }} / V$ where $\mathrm{V}_{\text {pore }}$ is the volume of the void-space and $V$ is the volume of the bulk material and was estimated by the dimensions of aerogel. $V_{\text {pore }}$ was estimated via $V_{\text {pore }}=V-V_{\mathrm{rGO}}$ ( $V_{\mathrm{rGO}}=m / d_{\mathrm{rGO}}$ where $d_{\mathrm{rGO}}=2 \mathrm{~g} \mathrm{~cm}^{3}$ is the density of $\mathrm{rGO}$ and $m$ is the mass of the bulk material) (Chen et al., 2013). 
TABLE 1 | Diamines used as gelators for rGO hydrogels.

\begin{tabular}{|c|c|c|c|}
\hline \multicolumn{2}{|r|}{ Aliphatics } & \multirow{2}{*}{$\begin{array}{r}\mathbf{p K a}_{\mathbf{1}} \\
11.9\end{array}$} & \multirow{2}{*}{$\begin{array}{l}\text { Solubility in water } \\
800 \mathrm{~g} \mathrm{~L}^{-1}\end{array}$} \\
\hline HDA & & & \\
\hline EDA & & 10.7 & Miscible \\
\hline TETA & & 9.9 & $100 \mathrm{~g} \mathrm{~L}^{-1}$ \\
\hline PXDA & & 9.2 & Miscible \\
\hline \multicolumn{4}{|c|}{ AROMATICS } \\
\hline PPD & & 6.2 & $40 \mathrm{~g} \mathrm{~L}^{-1}$ \\
\hline MDA & & 4.8 & $1 \mathrm{~g} \mathrm{~L}^{-1}$ \\
\hline OPD & & 4.7 & $<1 \mathrm{~g} \mathrm{~L}^{-1}$ \\
\hline DAB & & 3.6 & $<1 \mathrm{~g} \mathrm{~L}^{-1}$ \\
\hline
\end{tabular}

TABLE 2 | Bulk resistance (R), resistivity ( $\boldsymbol{\rho}$ ), dimensions, and density (d) of the rGO aerogels.

\begin{tabular}{lcccccc}
\hline rGO & $\begin{array}{c}\text { Mass } \\
(\mathbf{m g})\end{array}$ & $\begin{array}{c}\mathbf{d}_{\mathbf{r G O}}(\mathbf{m g} \\
\mathbf{c m} \mathbf{- 3})\end{array}$ & $\begin{array}{c}\mathbf{S}\left(\mathbf{m}^{\mathbf{2}}\right) \\
\left.\mathbf{( *}^{*} \mathbf{1 0}^{-\mathbf{4}}\right)\end{array}$ & $\mathbf{R}(\mathbf{k} \boldsymbol{\Omega})$ & $\boldsymbol{\rho}(\boldsymbol{\Omega} \mathbf{m})$ & Porosity $\%$ \\
\hline EDA & 20 & 12.9 & 3.1 & 6.0 & 620 & 99.4 \\
TETA & 20 & 22.0 & 1.8 & 3.6 & 200 & 98.9 \\
HDA & 21 & 21.0 & 2.0 & 40.0 & 2600 & 99.0 \\
PXDA & 22 & 22.0 & 2.0 & 1.6 & 106 & 98.9 \\
MDA & 26 & 65.0 & 0.8 & 2.0 & 52 & 96.8 \\
OPD & 16 & 12.8 & 2.5 & 0.3 & 29 & 99.4 \\
PPD & 20 & 21.0 & 3.1 & 1.2 & 124 & 99.4 \\
DAB & 21 & 12.0 & 3.6 & 0.3 & 25 & 99.4 \\
\hline
\end{tabular}

The thickness was measured to $0.5 \mathrm{~cm}$.

\section{RESULTS AND DISCUSSION}

\section{The Diamines}

Diamines selected for this study are divided in two groups: aliphatic and aromatic. The first group includes flexible ethylenediamine (EDA), 1,6 hexanediamine (HDA), triethylenetetramine (TETA), and a semi-flexible one, paraxylylenediamine (PXDA). The second group includes rigid aniline derivatives such as ortho-phenylenediamine (OPD), para-phenylenediamine (PPD), 3,3'-diaminobenzidine (DAB), and a semi-flexible one, 4,4'-methylene dianiline (MDA). The aliphatic diamines are classified as strong bases and strong nucleophiles, and they were water soluble (see Table 1). On the other hand, aromatic diamines are weak bases and poorly soluble in water. EDA (Kim et al., 2013) and PPD (Ma et al., 2012)

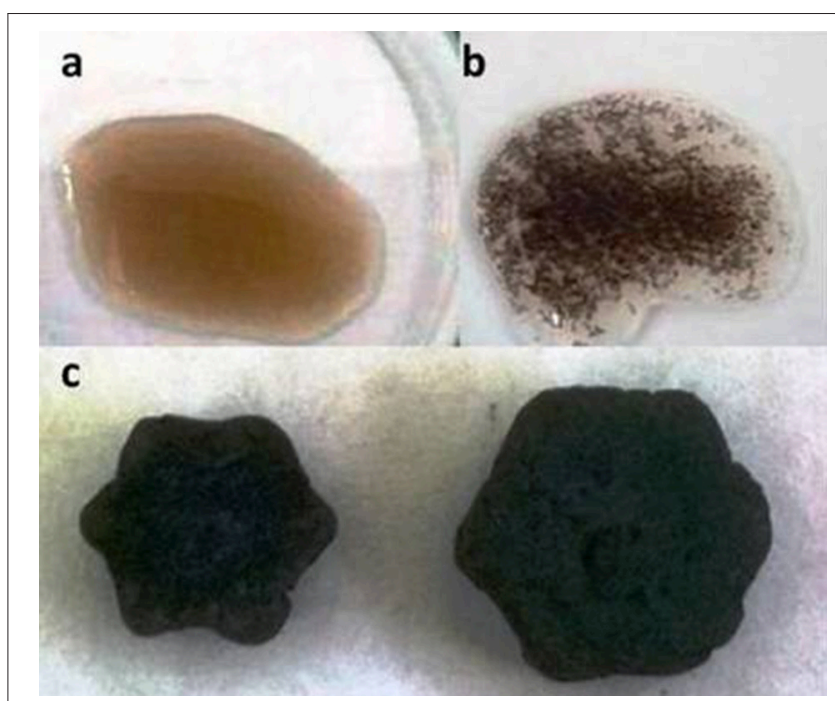

FIGURE 2 | The reaction mixture before (a) and after (b) the addition of TETA. (c) Well shaped hexagonal aerogels from the hydrothermal treatment of TETA with GO.

have been previously reported in the hydrothermal synthesis of graphene aerogels.

\section{Hydrogel Formation}

During hydrothermal process, after the addition of diamine, GO nanosheets agglomerated forming monolithic hydrogels as shown in Figure 1. With the exception of MDA, hydrogels had almost the same volume as the sol. Hydrogels with MDA underwent excessive shrinkage (about 50\% in linear dimensions). All hydrogels were washed extensively with water and acetone to remove unreacted diamines and ammonia, and they were dried to aerogels using freeze-drying (see Experimental Section).

Introducing diamines as gelation promoters has the dual advantage of using a single reagent both as a crosslinker of GO sheets, and as a reducing agent (Herrera-Alonso et al., 2007; Li et al., 2011; Ma et al., 2012; Kim et al., 2013). In that regard, nucleophilic attack of diamines to an epoxide group on the GO surface leads to fuctionalization of graphene with diamines, which in part is followed by a second nucleophilic attack, formation of an aziridine ring and elimination of $\mathrm{OH}$ as water; eventually, elimination of the aziridine ring itself leaves behind a double bond, in analogy to the mechanism suggested by Stankovich et al. for the reduction of GO by hydrazine (Stankovich et al., 2007).

Functionalization was evidenced by the presence of the diamine on the surface. Partial reduction was accompanied by enhanced conductivity and a drastic decrease of oxygen groups in the final product. The first remarkable difference between the aliphatic and aromatic diamines was observed directly after their addition in the GO dispersion: addition of aliphatic diamines resulted in immediate formation of GO nanosheet aggregates (see Figures 2a,b). On the other hand, aniline derivatives were dispersed slowly in the water phase, and GO aggregates were formed much later during the hydrothermal heating. 

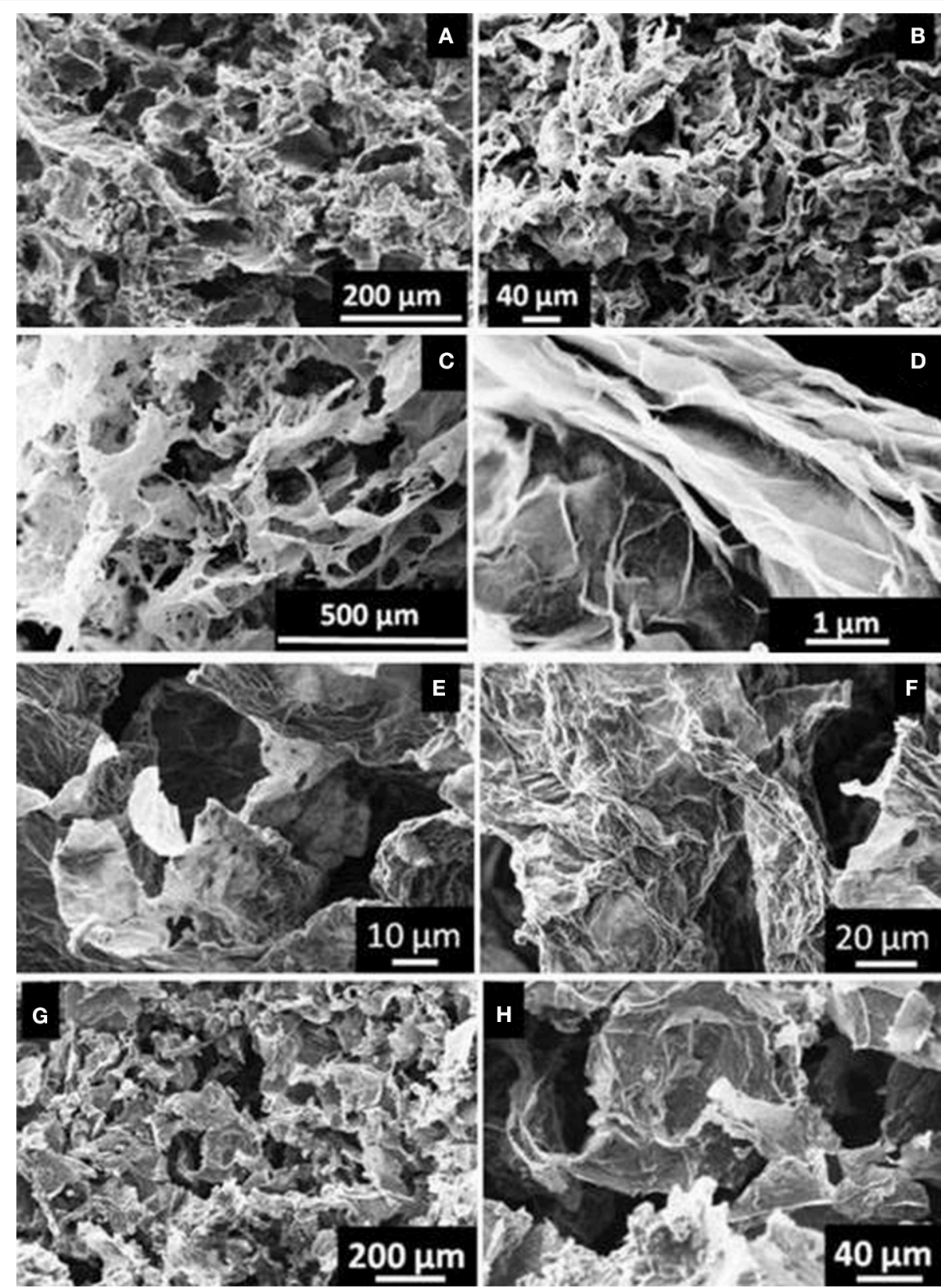

FIGURE 3 | SEM images of (a,b) rGO TETA, (c,d) rGOHDA, (e,f) rGO $\mathrm{rGDA}_{\text {, }}$ and $\mathbf{( g , \mathbf { h } ) \text { rGO }}$ PXDA aerogels.

During hydrothermal treatment, diamines function as crosslinkers leading to agglomeration of functionalized rGO in well-shaped black monolithic hydrogels. The density of the final aerogels ranged between 12 and $22 \mathrm{mg} \mathrm{cm}^{-3}$ (except for $\left.\mathrm{rGO}_{\mathrm{MDA}}\right)$. Direct aggregation of GO nanosheets via reaction of epoxy groups with aliphatic diamines, before reduction (which normally lead to graphitized species) led to the formation of well-shaped gels and aerogels that kept the dimensions of the mold as demonstrated in Figure 2c, with a hexagonal-shaped aerogel. Hydrothermal treatment of GO, under the same conditions, in the absence of diamine, led to the formation of
rGO aggregates, which after removal of water remained as dense powders.

\section{Characterization of Graphene Aerogels}

The mass of functionalized rGO aerogels ranged between 16 and $26 \mathrm{mg}$, while the porosity was estimated above $96 \%$ for all materials (see Table 2). The microstructure of final aerogels with aliphatic diamines consists of pores with diameter between 20 and $40 \mu \mathrm{m}$ as revealed by SEM (see Figure 3).

Similarly, GO nanosheets with aromatic diamines were partly reduced and functionalized leading to porous $3 \mathrm{D}$ monolithic 


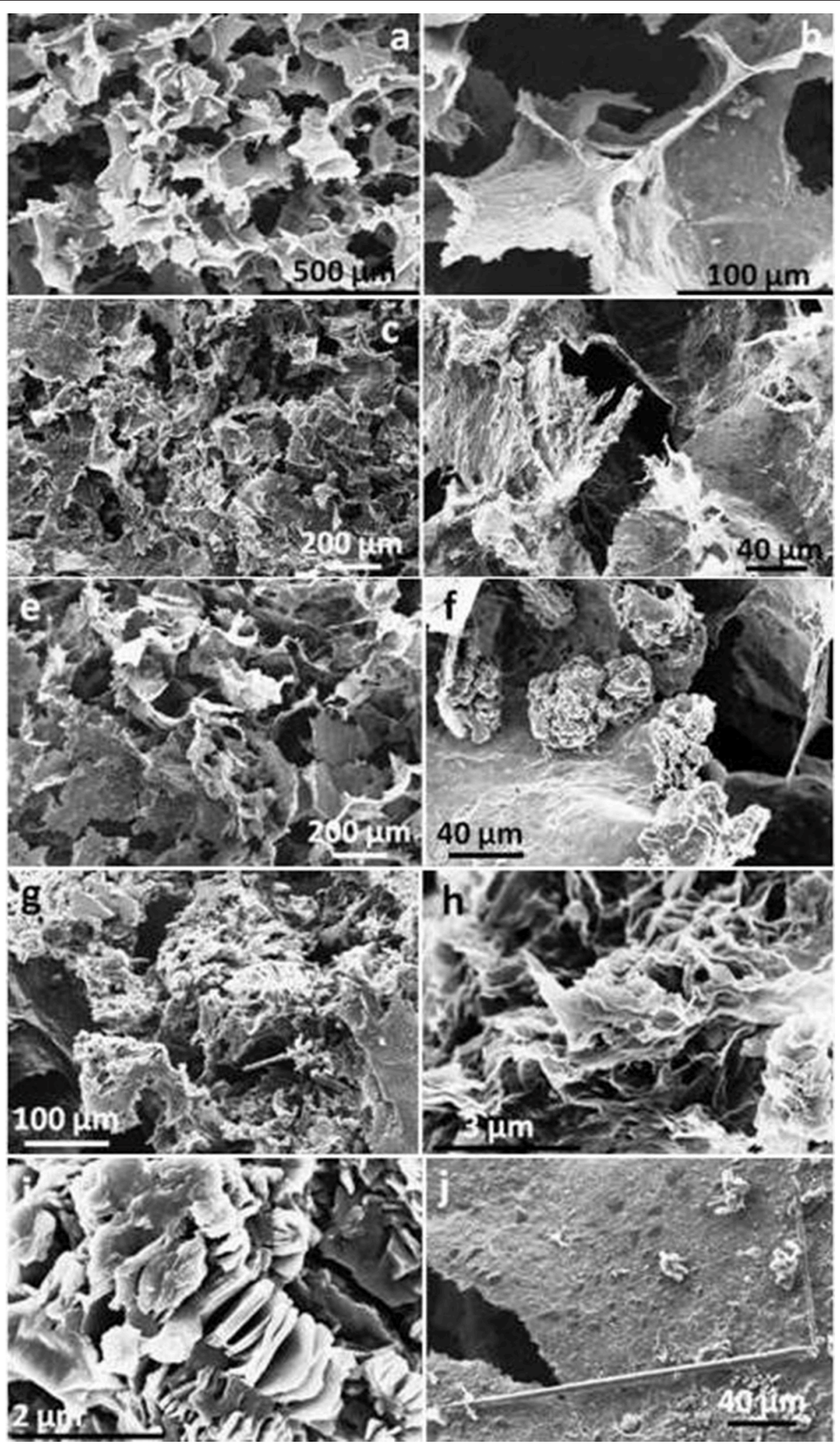

FIGURE 4 | SEM images of (a,b) rGOPPD, (c,d) rGO graphene surface in $r G O_{D A B}$ and $(\mathbf{J}) r_{\text {GOMDA }}$ aerogels. 


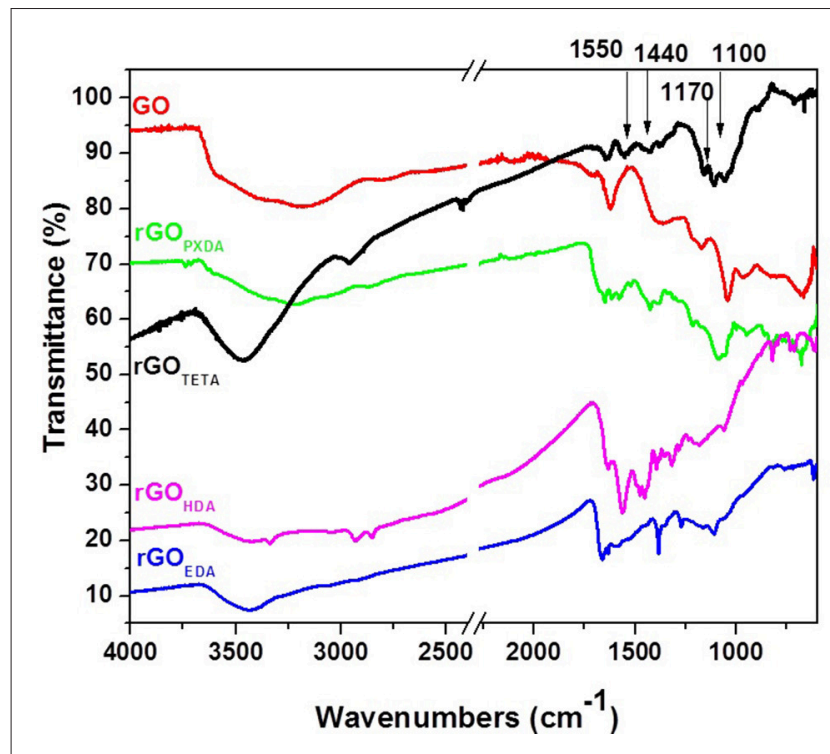

FIGURE 5 | FT-IR spectra of aerogels with aliphatic diamines.

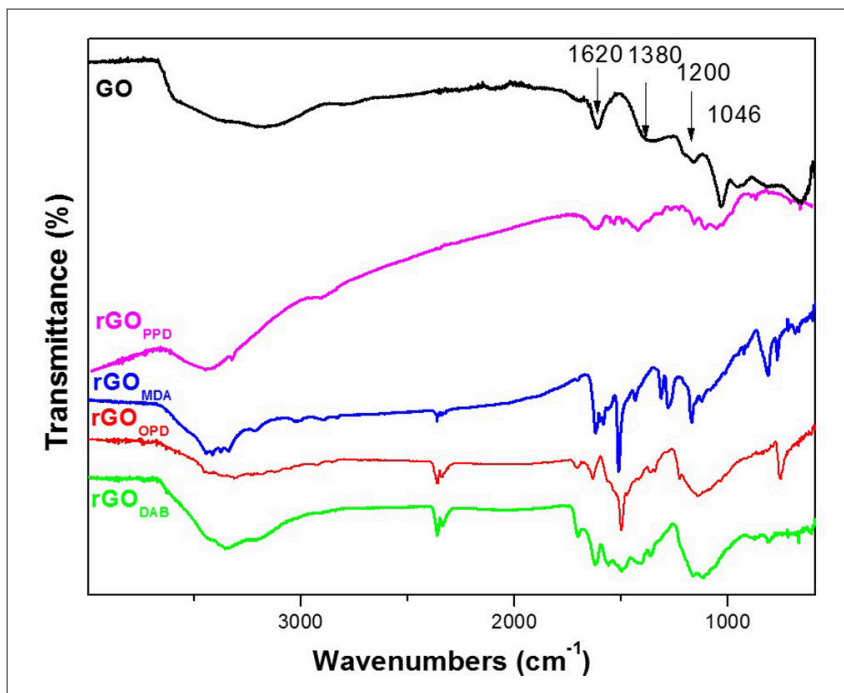

FIGURE 6 | FT-IR spectra of aerogels with aromatic diamines.

aerogels as shown in Figure 4. Here, pore sizes ranged from about 50 to $200 \mu \mathrm{m}$ in $\mathrm{rGO}_{\mathrm{PPD}}, \mathrm{rGO}_{\mathrm{OPD}}, \mathrm{rGO}_{\mathrm{DAB}}$ aerogels, while $\mathrm{rGO}_{\mathrm{MDA}}$ appeared to be more condensed without visible pores. The last two aerogels were randomly decorated with crystals of the diamines that were formed during synthesis, due to the very low solubility of MDA and DAB in water.

FT-IR spectra of all aerogels showed, in comparison with the spectrum of GO, a significant decrease of the characteristic GO bands, and appearance of new bands from the incorporated diamines (see Figures 5, 6). The spectrum of pure GO showed a broad band at $3,400-3,200 \mathrm{~cm}^{-1}$ due to the $\mathrm{OH}$ stretching vibration (carboxyl, hydroxyl groups, or intercalated water), a shoulder at $1,710 \mathrm{~cm}^{-1}$ (carboxylic $\mathrm{C}=\mathrm{O}$ stretch) and bands at $1,380 \mathrm{~cm}^{-1}$ (carboxylic O-H deformation vibration), $1,620 \mathrm{~cm}^{-1}$ (aromatic $\mathrm{C}=\mathrm{C}$ stretches) (Ma et al., 2012; Hu et al., 2013; Verma and Dutta, 2015), 1,200 $\mathrm{cm}^{-1}$ and $1,046 \mathrm{~cm}^{-1}$ [usually assigned to phenolic $\mathrm{C}-\mathrm{OH}$ stretches, as well as to epoxide (or alcoxy) stretches (Guo et al., 2009; Ma et al., 2012; Kellici et al., 2014)]. After reduction/functionalization, the bands assigned to oxygen groups at $1,046,1,200,1,710$, and $3,200-3,400 \mathrm{~cm}^{-1}$ were significantly reduced in the spectra of rGO's. Covalent grafting of TETA on graphenic layers was indicated by the appearance of characteristic peaks at 2,920, 2,850 $\mathrm{cm}^{-1}\left(-\mathrm{CH}_{2}\right.$ - stretch), $1,550 \mathrm{~cm}^{-1}(\mathrm{~N}-\mathrm{H}$ in plane scissoring vibration or bending), (Tang et al., 2014) $1,440 \mathrm{~cm}^{-1}\left(-\mathrm{CH}_{2}\right.$ - bend), $1,170 \mathrm{~cm}^{-1}$ and $1,100 \mathrm{~cm}^{-1}$ (C-N stretch). Similar spectroscopic evidence was recorded in other aerogels with aliphatic diamines as shown in Figure 5, indicating analogous covalent functionalization.

Regarding the FT-IR spectra of the aerogels with aromatic diamines a similar behavior is observed. The characteristic peaks of the oxygen groups were remarkably reduced in comparison with GO and several new peaks have been appeared indicating the presence of aromatic diamines in the aerogels. $\mathrm{rGO}_{\mathrm{PPD}}$ showed peaks at $3,445 \mathrm{~cm}^{-1}(\mathrm{OH}$ stretch $), 1,624 \mathrm{~cm}^{-1}(\mathrm{C}=\mathrm{C}$ bending), $1,536 \mathrm{~cm}^{-1}$ (bending vibration of $\mathrm{NH}$, indicating $-\mathrm{C}$ $\mathrm{NH}-\mathrm{C}$ - formation) (Tang et al., 2014), 1,500 $\mathrm{cm}^{-1}$ (phenyl ring vibration), 1,420 (C-N stretch), 1,100, $\mathrm{cm}^{-1}$ (-CO stretch), (Lu et al., 2014), and $873 \mathrm{~cm}^{-1}$ (CH non-planar ring vibrations; see Figure 6).

$\mathrm{rGO}_{\mathrm{MDA}}$ showed weak bands at $3,412 \mathrm{~cm}^{-1}\left(\mathrm{NH}\right.$ and $\mathrm{NH}_{2}$ stretch), $1,625 \mathrm{~cm}^{-1}\left(\mathrm{C}=\mathrm{C}\right.$ bending), $1,500 \mathrm{~cm}^{-1}$ (phenyl ring vibration), $1,230,1,160 \mathrm{~cm}^{-1}$ (C-N stretch), and $822 \mathrm{~cm}^{-1}$ ( $\mathrm{CH}$ non-planar ring vibrations). $\mathrm{rGO}_{\mathrm{DAB}}$ showed peaks at $3,352 \mathrm{~cm}^{-1}$ ( $\mathrm{NH}$ and $\mathrm{NH}_{2}$ stretch), $1,625 \mathrm{~cm}^{-1}$ ( $\mathrm{C}=\mathrm{C}$ bending or $\mathrm{NH}_{2}$ scissors), $1,573 \mathrm{~cm}^{-1}$ (in plane scissoring vibrations or bending), $1,500,1,480 \mathrm{~cm}^{-1}$ (phenyl ring vibration), $1,425 \mathrm{~cm}^{-1}$ (C-N stretch), $1,140 \mathrm{~cm}^{-1}$ (C-O stretch) (see Figure 6).

\section{Raman Spectroscopy}

Raman spectra of GO and rGO aerogels are shown in Figure 7. $\mathrm{D}\left(\mathrm{A}_{1 \mathrm{~g}}\right.$ symmetry mode) and $\mathrm{G}\left(\mathrm{E}_{2 \mathrm{~g}}\right.$ mode of the $\mathrm{sp}^{2}$ carbon atoms) (Chen et al., 2013) bands in the modified with aliphatic diamines rGO aerogels appeared as well-defined peaks at $\sim 1,350$ and $1,580 \mathrm{~cm}^{-1}$, respectively. The same bands in the GO precursor appeared at 1,366 and $1,600 \mathrm{~cm}^{-1}$. And the observed shift is attributed to the recovery of the hexagonal network of the carbon atoms with defects. The $\mathrm{G}$ band in some spectra appeared clearly asymmetric due to the contribution of D' band, while both $\mathrm{D}$ and $\mathrm{G}$ bands in $\mathrm{rGO}_{\mathrm{DAB}}, \mathrm{rGO}_{\mathrm{OPD}}$ aerogels are contaminated by signals probably due to the presence of aromatic diamines. The characteristic $I_{D} / I_{G}$ ratio $\left(I_{D}\right.$ and $I_{G}$ were measured from the peak height after baseline correction) was increased from 0.78 in pure GO to $0.86-1.19$, similarly to other rGO products in the literature (Stankovich et al., 2007; Pei and Cheng, 2012; Tang et al., 2014; Abdolhosseinzadeh et al., 2015; Bo et al., 2015; Ji et al., 2017). That change is usually attributed to the decrease of average size of $\mathrm{sp}^{2}$ domains, together with an increase of the number of those domains after reduction (Stankovich et al., 2007; Pei and Cheng, 2012; Chen et al., 2013; Feng et al., 2013). Moreover, the $2 \mathrm{D}$ band in all products appeared between 2,700 and $2,730 \mathrm{~cm}^{-1}$, while it was sharper and more intense in $\mathrm{rGO}_{\mathrm{TETA}}$ and $\mathrm{rGO}_{\mathrm{HDA}}$ 

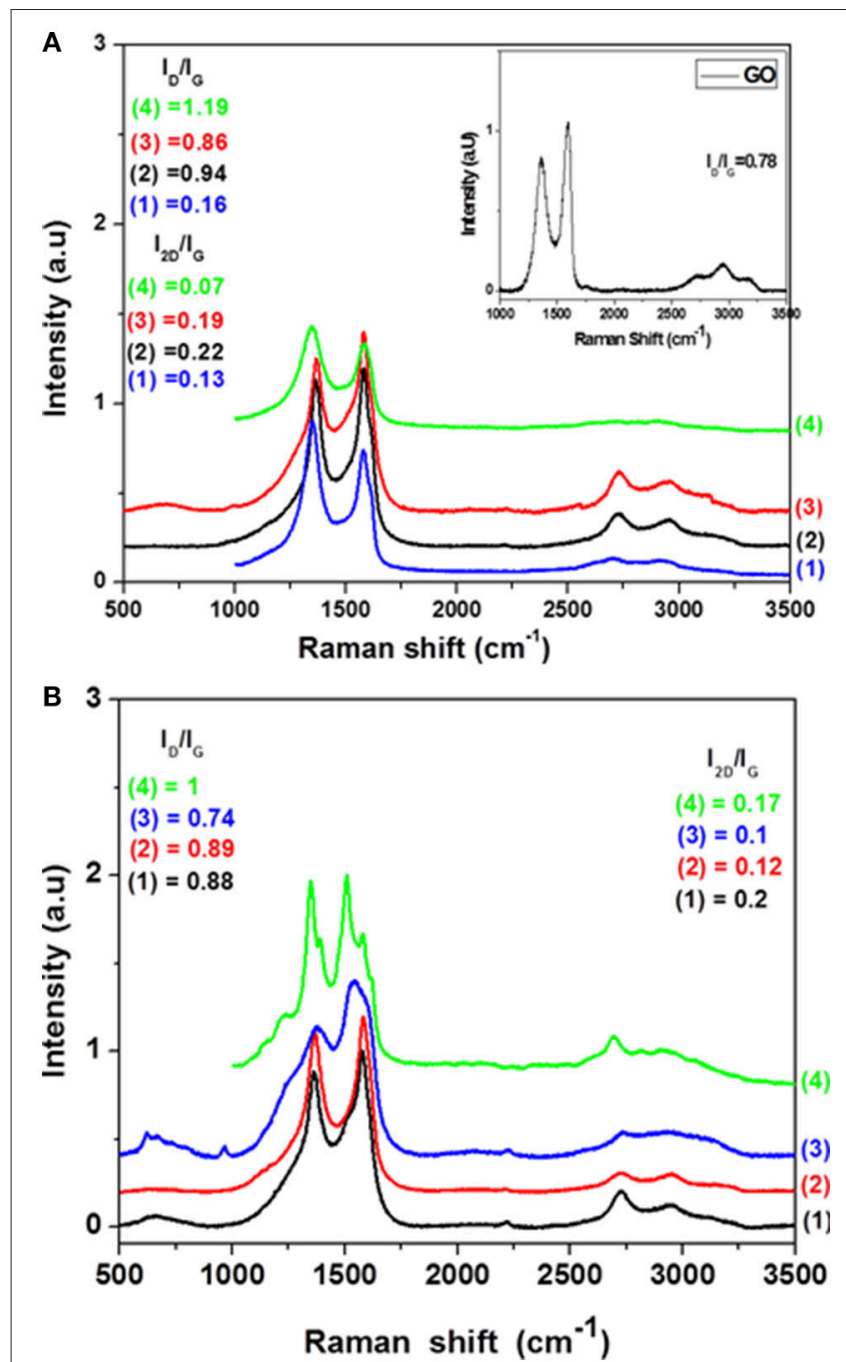

FIGURE 7 | Raman spectra of aerogels with (A) aliphatic diamines (1:rGOEDA, 2:rGOHDA, 3:rGOTETA, 4:rGOPXDA), (B) aromatic diamines (1:rGOPPD, 2:rGO $\left.\mathrm{MDA}, 3: \mathrm{rGO}_{\mathrm{DAB}}, 4: \mathrm{rGO}_{\mathrm{OPD}}\right)$.

indicating a higher degree of aromatization in those products, in accordance with the results of TG analysis as shown below. Similarly, in rGO aerogels modified with aromatic diamines, the $\mathrm{I}_{\mathrm{D}} / \mathrm{I}_{\mathrm{G}}$ ratio ranged between 0.74 and 1 . The $2 \mathrm{D}$ band here appeared near $2,730 \mathrm{~cm}^{-1}$ (except in $\mathrm{rGO}_{\mathrm{OPD}}$ where it appeared at 2,692 $\mathrm{cm}^{-1}$ ), while it was sharper and more intense in $\mathrm{rGO}_{\mathrm{PPD}}$ and $\mathrm{rGO}_{\mathrm{OPD}}$ indicating an analogous higher degree of aromatization of those products.

\section{TG Analysis}

Thermogravimetric analysis of GO showed a 5\% mass loss up to $100^{\circ} \mathrm{C}$ due to removal of water entrapped between GO nanosheets and a $35 \%$ mass loss closer to $200^{\circ} \mathrm{C}$, which is attributed to pyrolytic removal of oxygen-containing groups (epoxides, carboxyl, and hydroxyl groups). The remaining carbon material was removed by oxidation completely by $500^{\circ} \mathrm{C}$

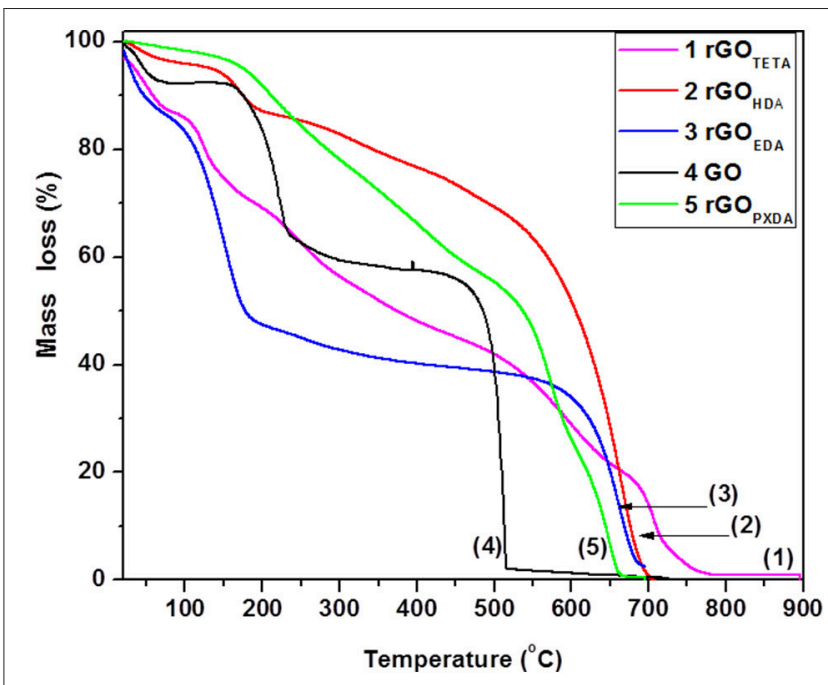

FIGURE 8 | TG analysis of aerogels with aliphatic diamines and GO.

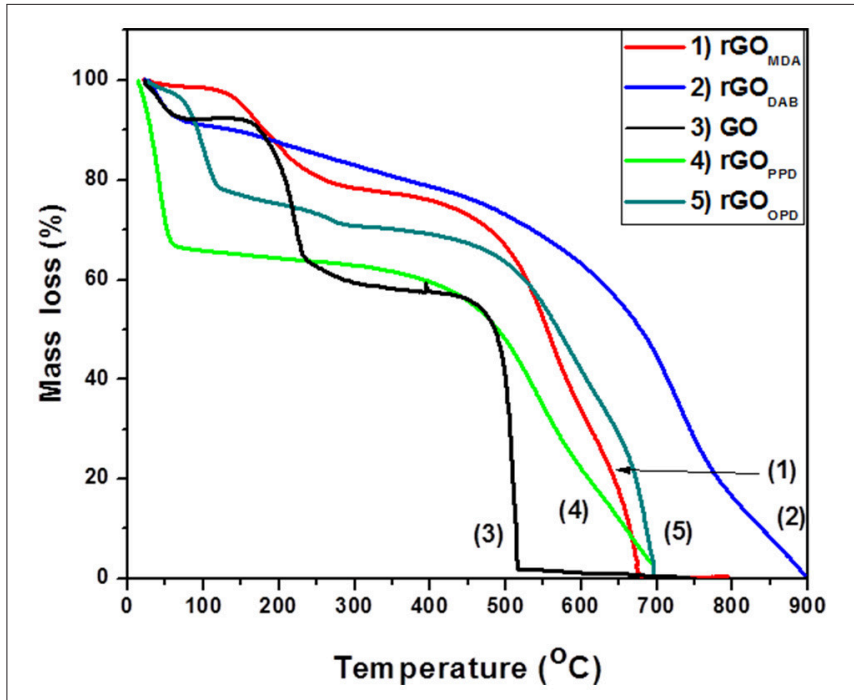

FIGURE 9 | TG analysis of aerogels with aromatic diamines and GO.

(see Figure 8, line 4) (Kuila et al., 2008; Abdolhosseinzadeh et al., 2015).

Thermographs of rGO aerogels with aliphatic diamines, as shown in Figure 8, showed a mass loss between 30 and $60 \%$, over $600^{\circ} \mathrm{C}$, attributed to carbon oxidation, indicating enhanced thermal stability of rGO nanosheets, in comparison with GO, due to the partial aromatization during hydrogel formation. Furthermore, $\mathrm{rGO}_{\mathrm{EDA}}$ showed a mass loss of $45 \%$ between 100 and $200^{\circ} \mathrm{C}$, while the other rGO loose less mass, gradually between 100 and $550^{\circ} \mathrm{C}$. This is possibly attributed to the removal of diamine and oxygen containing groups. HDA appeared to be the most effective in reduction of GO, since $\mathrm{rGO}_{\mathrm{HDA}}$ was the most thermally stable (a property that correlates with aromaticity), in accordance with its superior basicity/nucleophilicity (see Table $\mathbf{1}, \mathrm{pK}_{\mathrm{a}}$ values). At the opposite 


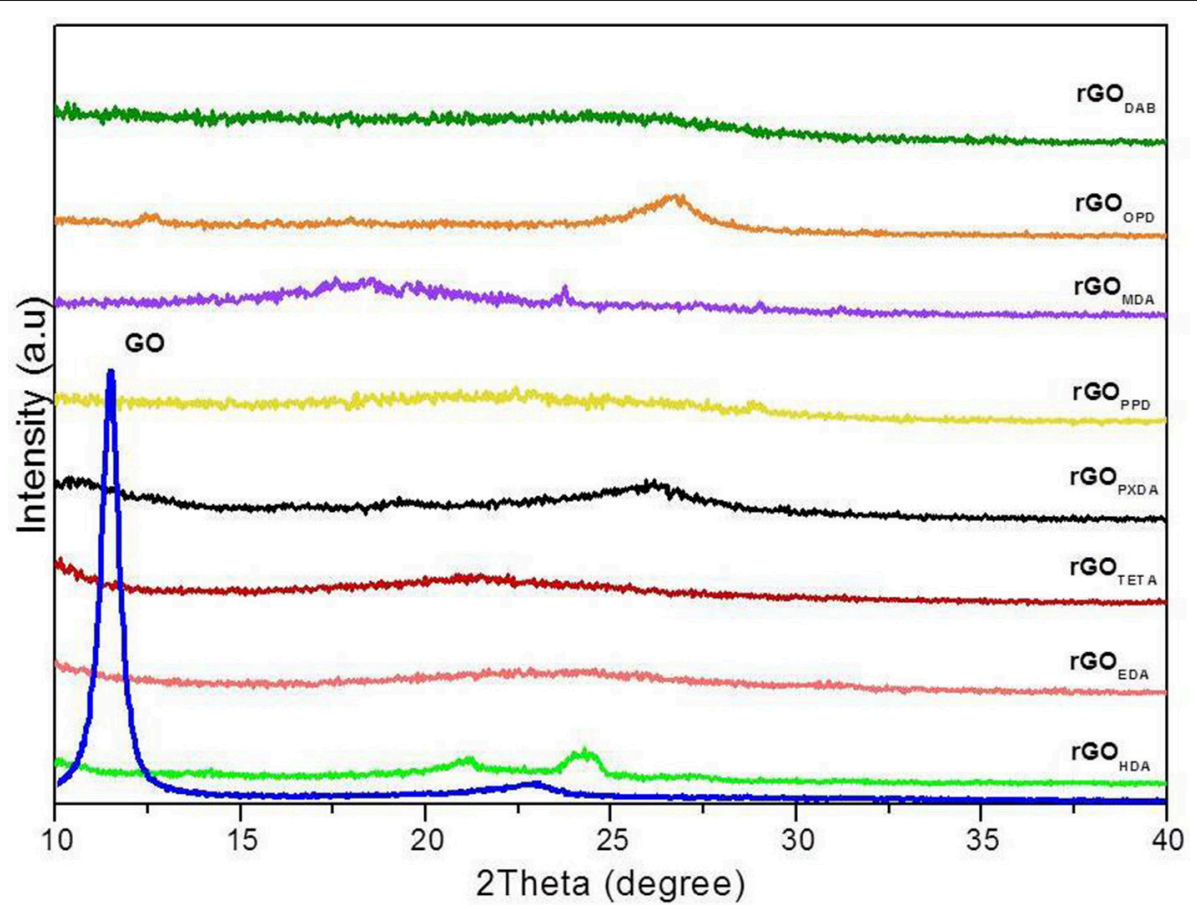

FIGURE 10 | XRD patterns of $r G O$ aerogels and GO.

PXDA, the weakest base/nucleophile, was the less effective in reduction of GO, producing less thermally stable $\mathrm{rGO}_{\mathrm{PXDA}}(\mathrm{He}$ et al., 2011).

The results from TGA of $\mathrm{rGO}$ aerogels with aromatic diamines are shown in Figure 9. $\mathrm{rGO}_{\mathrm{PPD}}$, showed a 35\% mass loss below $100^{\circ} \mathrm{C}$ (water removal, which appeared in most aerogels) and almost all the rest material (PPD and carbon mass) was removed between 500 and $700^{\circ} \mathrm{C}$. $\mathrm{rGO}_{\mathrm{OPD}}$ and $\mathrm{rGO}_{\mathrm{MDA}}$ showed similar behavior and lost about $70 \%$ between 500 and $700^{\circ} \mathrm{C}$ showing slightly more effective reduction. $\mathrm{rGO}_{\mathrm{DAB}}$ nanosheets were the most thermally stable and consequently most aromatic material since a $60 \%$ was removed above $700^{\circ} \mathrm{C}$. Comparing thermal stabilities of the four aerogel's nanosheets with aromatic diamines, it is observed that DAB was the best reducing agent, although it was the weakest base. Then, OPD and MDA followed and finally PPD, the strongest base (according to the $\mathrm{pKa}$ values), was the less effective reducing agent. That behavior could be attributed, either to the excess amino groups of $\mathrm{DAB}$, or the flexibility of MDA due to central aliphatic carbon that could affect the reduction mechanism, e.g., by facilitating aziridine formation or the elimination that follows nucleophilic addition of the diamine.

Figure 10 shows the XRD patterns of GO, and rGO aerogels. GO showed an intense peak at $2 \theta=11.4^{\circ}$, which corresponds to an interlayer distance of $0.77 \mathrm{~nm}$, due to absorbed water molecules between graphene layers and the existence of oxygen functional groups (Ma et al., 2012; Bo et al., 2015). The XRD patterns of $\mathrm{rGO}$ aerogels, showed absence of any intense characteristic peak, which is an indication of the effective exfoliation of rGO nanosheets. $\mathrm{rGO}_{\mathrm{OPD}}$ and $\mathrm{rGO}_{\mathrm{PXDA}}$ aerogels showed a weak broad peak at $2 \theta=26.7^{\circ}$ and $26.4^{\circ}$ respectively, which attributed to graphitic structure $(\mathrm{d}=0.34 \mathrm{~nm})$. The other aerogels showed very weak broad graphite (002) peaks with $2 \theta=21-24^{\circ}$, which corresponds to an interlayer spacing of $0.43-0.36 \mathrm{~nm}$ and is due to disordered stacking of the reduced graphene sheets (Bourlinos et al., 2003; Chen et al., 2013; Fan et al., 2013; Hu et al., 2013; Lan et al., 2016).

\section{Electrical Conductivity}

It is known that rGO aerogels are electrically conductive, which is a property that is recovered only after reduction and partial aromatization of GO (Ma et al., 2012; Nardecchia et al., 2013; Li et al., 2014; Jun et al., 2015). However, conductivity is not always the same and depends basically on the degree of reduction and restoration of the aromatic system as well as the interconnections between the rGO nanosheets.

To have a representative image of the electrical conductivity of aerogels, bulk electric resistance was measured instead of sheet resistance, which is preferable mainly in films, thus avoiding problems with the roughness and the porosity of the aerogel surfaces. Bulk resistance and resistivity of rGO aerogels are shown in Table 2. Although the resistivity values vary, there is a clear trend of aromatic diamines to reduce aerogel resistivity more efficiently in comparison with aliphatic diamines (Ma et al., 2012). Considering that electrical resistance of a graphene superstructure is located in the interconnections between graphene nanosheets, the reduced resistivity of the doped with aromatic diamines aerogels could be attributed to a positive role of the aromatic molecules as conductive pathways in 


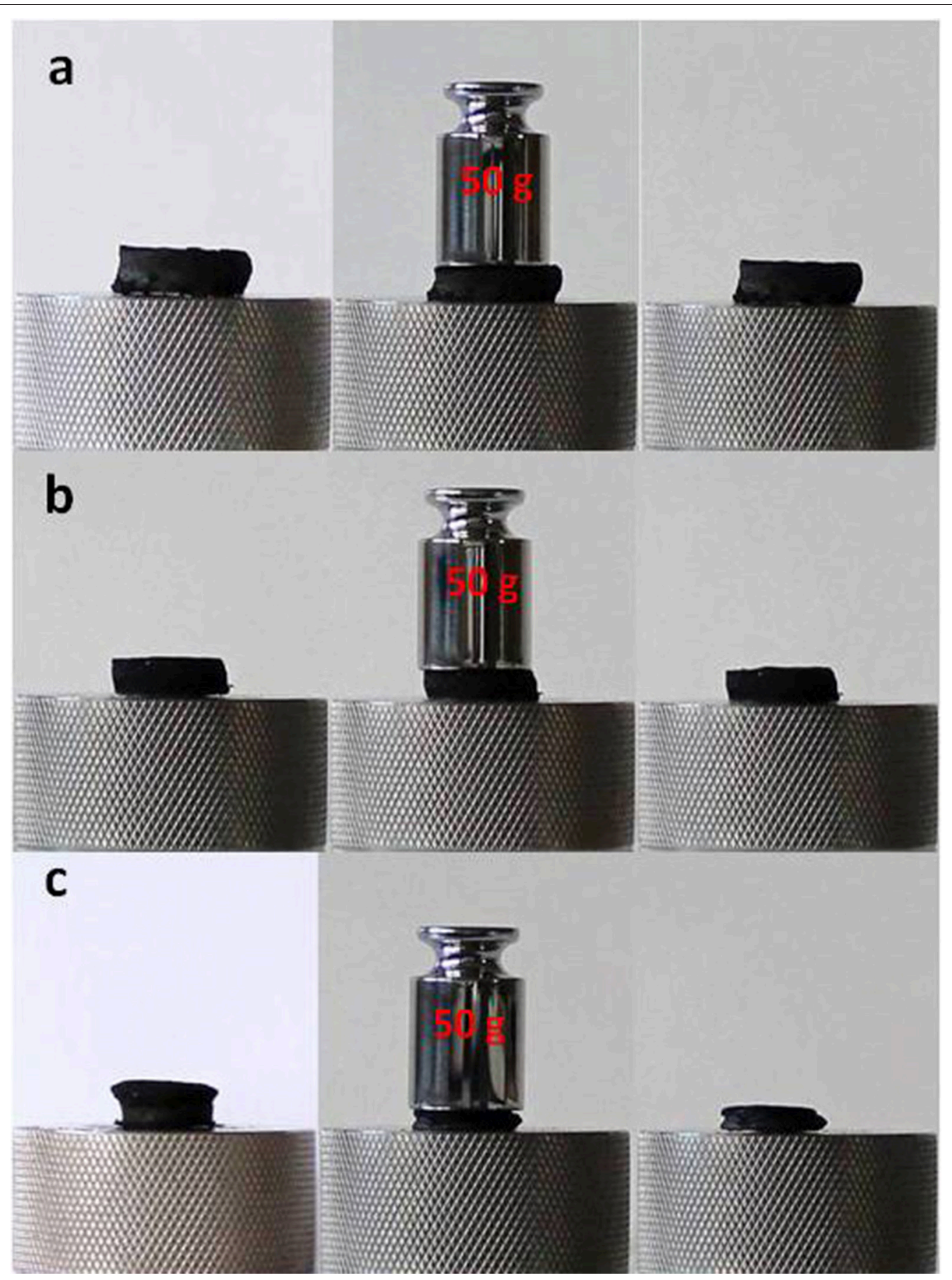

FIGURE 11 | The compression of (a) $\mathrm{rGO}_{\mathrm{OPD}}$ (b) rGOHDA (c) rGOTETA aerogel cylinders after the placement of a $50 \mathrm{~g}$ standard precision weight.

the interconnections. Furthermore, aromatic molecules could be placed as a bridge connecting graphene nanosheets, multiply the number of interconnections and thus decreasing the resistivity.

In contrast to their ultralight weight, graphene aerogels showed an outstanding mechanical strength as expressed by their ability to support loads about 2,500 times heavier than their own weight, without signs of deformation. As shown in Figure 11a, the placement of a $50 \mathrm{~g}$ standard weight on the top of $\mathrm{rGO}_{\mathrm{OPD}}$ aerogel cylinder, resulted in a compression of about 25\%. After the removal of the weight, $\mathrm{rGO}_{\mathrm{OPD}}$ aerogel cylinder recovered the initial thickness. Similar elastic compression was observed with all aerogels with aromatic diamines.

The aerogels with aliphatic diamines, under the same conditions, showed a lower-about 11\%-but inelastic 
compression, as shown in Figure $\mathbf{1 1 b}$ and $\mathrm{rGO}_{\mathrm{HDA}}$. The other aerogels with aliphatic diamines showed similar behavior, with the exception of $\mathrm{rGO}_{\mathrm{TETA}}$, which was compressed, also inelastic, about 50\%, under the same conditions (see Figure 11c).

\section{CONCLUSION}

GO was hydrothermally reduced and subsequently functionalized with aliphatic and aromatic diamines, forming well-defined 3D monolithic aerogels, with remarkable mechanical strength. This study has shown that aromatic diamines improved the conductivity of aerogels, while porosity and density were not seriously affected by the diamine structure or solubility. Amongst aliphatic diamines, the most nucleophilic one (HDA) was also the most effective in reducing GO, while

\section{REFERENCES}

Abdolhosseinzadeh, S., Asgharzadeh, H., and Kim, H. S. (2015). Fast and fully-scalable synthesis of reduced graphene oxide. Sci. Rep. 5:10160. doi: 10.1038/srep10160

Bo, Z., Shuai, X., Mao, S., Yang, H., Qian, J., Chen, J., et al. (2015). Green preparation of reduced graphene oxide for sensing and energy storage applications. Sci. Rep. 4:4684. doi: 10.1038/srep04684

Bourlinos, A. B., Gournis, D., Petridis, D., Szabo, T., Szeri, A., and Dekany, I. (2003). Graphite oxide: chemical reduction to graphite and surface modification with primary aliphatic amines and amino acids. Langmuir 19:6050. doi: 10.1021/la026525h

Brodie, B. C. (1859). On the atomic weight of Graphite. Philos. Trans. R. Soc. Lond. 149, 249-259. doi: 10.1098/rstl.1859.0013

Chen, D., Feng, H., and Li, J. (2012). Graphene oxide: preparation, functionalization, and electrochemical applications. Chem. Rev. 112, 6027-6053. doi: 10.1021/cr300115g

Chen, M., Zhang, C., Li, X., Zhang, L., Ma, Y., Zhang, L., et al. (2013). A one-step method for reduction and self-assembling of graphene oxide into reduced graphene oxide aerogels. J. Mater. Chem. A 1, 2869-2877. doi: $10.1039 / \mathrm{c} 2 \mathrm{ta} 00820 \mathrm{c}$

Chua, C. K., and Pumera, M. (2014). Chemical reduction of graphene oxide: a synthetic chemistry viewpoint. Chem. Soc. Rev. 43, 291-312. doi: 10.1039/C3CS60303B

Compton, O. C., and Nguyen, S. T. (2010). Graphene oxide, highly reduced graphene oxide, and graphene: versatile building blocks for carbon-based materials. Small 6, 711-723. doi: 10.1002/smll.200901934

Fan, Z., Tng, D. Z., Nguyen, S. T., Feng, J., Lin, C., Xiao, P., et al. (2013). Morphology effects on electrical and thermal properties of binder less graphene aerogels. Chem. Phys. Lett. 561-562, 92-96. doi: 10.1016/j.cplett.2013.01.033

Feng, H., Cheng, R., Zhao, X., Duan, X., and Li, J. (2013). A low-temperature method to produce highly reduced graphene oxide. Nat. Commun. 4, 1537-1539. doi: 10.1038/ncomms2555

Guo, H. L., Wang, X. F., Qian, Q. Y., Wang, F. B., and Xia, X. H. (2009). A Green approach to the synthesis of graphene nanosheets. ACS Nano 3, 2653-2659. doi: $10.1021 / \mathrm{nn} 900227 \mathrm{~d}$

He, G., Chen, H., Zhu, J., Bei, F., Sun, X., and Wang, X. (2011). Synthesis and characterization of graphene paper with controllable properties via chemical reduction. J. Mater. Chem. 21, 14631-14638. doi: 10.1039/c1jm12393a

He, H., Klinowski, J., Forster, M., and Lerf, A. (1998). A new structural model for graphite oxide. Chem. Phys. Lett. 287, 53-56. doi: 10.1016/S0009-2614(98)00144-4

Herrera-Alonso, M., Abdala, A. A., McAllister, M. J., Aksay, I. A., and Prud'homme, R. K. (2007). Intercalation and stitching of graphite oxide with diaminoalkanes, Langmuir 23, 10644-10649. doi: 10.1021/la0633839

Hofmann, U., and Konig, E. (1937). Untersuchungen über Graphitoxyd. Z. Anorg. Allg. Chem. 234, 311-336. doi: 10.1002/zaac.19372340405 among aromatic diamines, a similar correlation was not observed since $\mathrm{DAB}$, the less nucleophilic one, was the most effective in reducing GO. Finally, it is concluded that aliphatic and aromatic diamines are excellent gelation promoters forming stable well-defined aerogel monoliths.

\section{AUTHOR CONTRIBUTIONS}

All authors listed have made a substantial, direct and intellectual contribution to the work, and approved it for publication.

\section{ACKNOWLEDGMENTS}

The authors thank Dr. N. Leventis for his critical reading of the manuscript and his suggestions for improvement.

Hontoria-Lucas, C., López-Peinado, A. J., López-González, J. D., Rojas-Cervantes, M. L., and Martín-Aranda, R. M. (1995). Study of oxygen-containing groups in a series of graphite oxides: physical and chemical characterization. Carbon 33, 1585-1592. doi: 10.1016/0008-6223(95)00120-3

Hu, H., Zhao, Z., Wan, W., Gogotsi, Y., and Qiu, J. (2013). Ultralight and highly compressible graphene aerogels. Adv. Mater. 25, 2219-2223. doi: 10.1002/adma.201204530

Hummers, W. S., and Offeman, R. E. (1958). Preparation of graphitic oxide. J. Am. Chem. Soc. 80:1339. doi: 10.1021/ja01539a017

Ji, X., Song, Y., Han, J., Ge, L., Zhao, X., Xu, C., et al. (2017). Preparation of a stable aqueous suspension of reduced graphene oxide by a green method for applications in biomaterials. J. Colliod Interface Sci. 497, 317-324. doi: 10.1016/j.jcis.2016.09.049

Jun, Y. S., Sy, S., Ahn, W., Zarrin, H., Rasen, L., Tjandra, R., et al. (2015). Highly conductive interconnected graphene foam based polymer composite. Carbon 95, 653-658. doi: 10.1016/j.carbon.2015.08.079

Kellici, S., Acord, J., Ball, J., Reehal, H. S., Morgan, D., and Saha, B. (2014). A single rapid route for the synthesis of reduced graphene oxide with antibacterial activities. RSC Adv. 4, 14858-14861. doi: 10.1039/C3RA47573E

Kim, F., Cote, L. J., and Huang, J. (2010). Graphene oxide: surface activity and two-dimensional assembly. Adv. Mater. 22, 1954-1958. doi: 10.1002/adma.200903932

Kim, N. H., Kuila, T., and Lee, J. H. (2013). Simultaneous reduction, functionalization and stitching of graphene oxide with ethylenediamine for composites application. J. Mater. Chem. A 1, 1349-1358. doi: 10.1039/C2TA00853 J

Kuila, T., Bose, S., Khanra, P., Mishra, A. K., Kim, N. H., and Lee, J. H. (2008). A green approach for the reduction of graphene oxide by wild carrot root. Carbon 50, 914-921. doi: 10.1016/j.carbon.2011.09.053

Lan, Y., Jin, B., Deng, J., Luo, Y. (2016). Graphene/nickel aerogel: an effective catalyst for the thermal decomposition of ammonium perchlorate. RSC Adv. 6, 82112-82117. doi: 10.1039/C6RA15661D

Lerf, A., He, H., Forster, M., and Klinowski, J. (1998). Structure of graphite oxide revisited. J. Phys. Chem. B 102, 4477-4482. doi: 10.1021/jp97 31821

Li, C., and Shi, G. (2012). Three-dimensional graphene architectures. Nanoscale 4:5549. doi: $10.1039 / \mathrm{c} 2 \mathrm{nr} 31467 \mathrm{c}$

Li, C., and Shi, G. (2014). Functional gels based on chemically modified graphenes. Adv. Mater. 26, 3992-4012. doi: 10.1002/adma.201306104

Li, W., Tang, X. Z., Bin Zhang, H., Jiang, Z. G., Yu, Z. Z., Du, X. S., et al. (2011). Simultaneous surface functionalization and reduction of graphene oxide with octadecylamine for electrically conductive polystyrene composites. Carbon 49 , 4724-4730. doi: 10.1016/j.carbon.2011.06.077

Li, Y., Samad, Y. A., Polychronopoulou, K., Alhassan, S. M., and Liao, K. (2014). Highly electrically conductive nanocomposites based on polymer-infused graphene sponges. Sci. Rep. 4:4652. doi: 10.1038/srep 04652 
Liu, F., and Seo, T. S. (2010). A controllable self-assembly method for large-scale synthesis of graphene sponges and free-standing graphene films. Adv. Funct. Mater. 20, 1930-1936, doi: 10.1002/adfm.201000287

Lu, Y., Huang, Y., Zhang, F., Zhang, L., Yang, X., Zhang, T., et al. (2014). Functionalized graphene oxide based on p-phenylenediamine as spacers and nitrogen dopants for high performance supercapacitors. Chin. Sci. Bull. 59, 1809-1815. doi: 10.1007/s11434-014-0297-3

Ma, H. L., Zhang, H. B., Hu, Q. H., Li, W. J., Jiang, Z. G., Yu, Z. Z., et al. (2012). Functionalization and reduction of graphene oxide with p-phenylene diamine for electrically conductive and thermally stable polystyrene composites. ACS Appl. Mater. Interfaces 4, 1948-1953. doi: 10.1021/am201654b

Mao, S., Pu, H., and Chen, J. (2012). Graphene oxide and its reduction: modeling and experimental progress. RSC Adv. 2, 2643-2662. doi: 10.1039/c2ra00663d

Nardecchia, S., Carriazo, D., Ferrer, M. L., Gutierrez, M. C., and del Monte, F. (2013). Three dimensional macroporous architectures and aerogels built of carbon nanotubes and/or graphene: synthesis and applications. Chem. Soc. Rev. 42, 794-830. doi: 10.1039/C2CS35353A

Novoselov, K. S., Geim, A. K., Morozov, S. V., Jiang, D., Zhang, Y., Dubonos, S. V., et al. (2004). Electric field effect in atomically thin carbon films. Science 306:666. doi: $10.1126 /$ science. 1102896

Park, S., and Ruoff, R. S. (2009). Chemical methods for the production of graphenes. Nat. Nanotechnol. 4, 217-224. doi: 10.1038/nnano.2009.58

Pei, S., and Cheng, H. M. (2012). The reduction of graphene oxide. Carbon 50, 3210-3228. doi: 10.1016/j.carbon.2011.11.010

Stankovich, S., Dikin, D. A., Piner, R. D., Kohlhaas, K. A., Kleinhammes, A., Jia, Y., et al. (2007). Synthesis of graphene-based nanosheets via chemical reduction of exfoliated graphite oxide. Carbon 45, 1558-1565. doi: 10.1016/j.carbon.2007.02.034

Staudenmaier, L. (1898). Verfahren zur darstellung der graphitsaure. Ber. Dtsch. Chem. Ges. 31, 1481 -1487. doi: 10.1002/cber.18980310237
Sun, H., Xu, Z., and Gao, C. (2013). Multifunctional, ultra-flyweight, synergistically assembled carbon aerogels. Adv. Mater. 25, 2554-2560. doi: 10.1002/adma.201204576

Tang, G., Jiang, Z. G., Li, X., Zhang, H. B., Dasari, A., and Yu, Z. Z. (2014). Three-dimensional graphene aerogels and their electrically conductive composites. Carbon 77, 592-599. doi: 10.1016/j.carbon.2014. 05.063

Verma, S., and Dutta, R. K. (2015). A facile method of synthesizing ammonia modified graphene oxide for efficient removal of uranyl ions from aqueous medium. RSC Adv. 5, 77192-77203. doi: 10.1039/C5RA 10555B

Zhang, L., Chen, G., Hedhili, M. N., Zhanga, H., and Wang, P. (2012). Three-dimensional assemblies of graphene prepared by a novel chemical reduction-induced self-assembly method Nanoscale 4, 7038-7045. doi: 10.1039/C2NR32157B

Zhu, Y., Murali, S., Cai, W., Li, X., Suk, J. W., Potts, J. R., et al. (2012). Graphene and graphene oxide: synthesis, properties, and applications. Adv. Mater. 22, 3906-3924. doi: 10.1002/adma.201001068

Conflict of Interest Statement: The authors declare that the research was conducted in the absence of any commercial or financial relationships that could be construed as a potential conflict of interest.

Copyright (๑) 2018 Vrettos, Karouta, Loginos, Donthula, Gournis and Georgakilas. This is an open-access article distributed under the terms of the Creative Commons Attribution License (CC BY). The use, distribution or reproduction in other forums is permitted, provided the original author(s) and the copyright owner are credited and that the original publication in this journal is cited, in accordance with accepted academic practice. No use, distribution or reproduction is permitted which does not comply with these terms. 